\title{
Foreword
}

\section{Phenotypic and Developmental Plasticity}

One of the most exciting areas of research in contemporary biology concerns the attempt to understand the basis of variation in traits exhibited by cells, groups of cells and individual organisms. Until recently, it was taken for granted that the only significant source of variation for evolution, and by implication for all of biology, was genetic variation. Because it was assumed that non-genetic variation was of no relevance for evolutionary change, this attitude persisted in spite of the long-standing evidence for the existence of variation due to environmental causes or alternative developmental pathways. It is becoming increasingly clear that such a viewpoint is no longer tenable. Indeed, what is referred to as the plasticity of the phenotype - multiplicity of biological form and function against a constant genetic background - is coming to occupy center stage with regard to a large number of major issues in biology.

The existence of plasticity in the development and expression of phenotype has ramifications for evolutionary theory, causing a rethinking of some of the premises of the currently prevailing neoDarwinian synthesis. Theoretical understanding of the genotype-phenotype relationship, the potential of developmental mechanisms to generate novel phenotypes, and notions of robustness and evolvability of development, increasingly invoke plasticity as a fundamental property of living systems. Although plasticity has a longer history in the behavioural sciences, it is gaining new ground in this field as well, in considerations of development and evolution of behaviour.

Biological research is increasingly adopting approaches based on the physical sciences. This is true not just at the molecular level, where it has long been commonplace, but also at multiple scales and different levels of integration. This has had a particular impact on empirical and theoretical studies of the capacity to vary - or plasticity - that organisms display. At least three factors seem to be responsible for this. First, the concepts of noise and stochasticity are central to understanding how genetic information gets translated into the synthesis of proteins. Second, developmental noise constitutes an important source of biological variation between individuals of the same genetic makeup. And third, our understanding of the generation of shape and form has been enriched by physical models that combine what is known about gene regulation with the treatment of early-stage embryos and organ primordia as 'soft condensed matter.' The realistic hope is that ultimately an integrated approach to developmental biology will yield valuable insights into the phenotypic options open to living systems, options that are in some sense generic - that is, are not uniquely tied to genetic makeup. The approach would make full use of a 'physics way' of looking at systems ranging from groups of cells to groups of multicellular individuals.

Based on both the increasing interest in plasticity and the new experimental and theoretical approaches to it, the two of us thought it worthwhile to explore the possibility of bringing together workers in different areas of biology who would share their knowledge about the variety of ways in which plasticity is central to biological function and form. An equally important purpose that we had in mind was to expose talented students to these concepts.

With the aim of focusing attention on these issues, a Discussion Meeting on the theme "Phenotypic and Developmental Plasticity" was held at Estuary Island, Trivandrum, from 16 to 20 December 2007. This special issue of Journal of Biosciences contains articles based on most of the presentations made at the meeting as well as one on a similar theme solicited afterward. In preparing their texts, the participantauthors have made use of responses that immediately followed their oral presentations as well as written opinions elicited from reviewers, some of whom were speakers too. It is hoped that this has given some degree of coherence to this journal issue. We are grateful to all the contributors and apologize to them, as well as to our readers, for the length of time it has taken to bring out the issue. Fortunately, in line with Journal of Biosciences policy, all the articles that were received earlier have been made available on the Web site of the journal, where they continue to be freely accessible (http://www.ias.ac.in/jbiosci). 
In ending, we express our thanks to the Indian Academy of Sciences, Bangalore, the Jawaharlal Nehru Centre for Advanced Scientific Research, Bangalore, the Rajiv Gandhi Centre for Biotechnology, Trivandrum, the Indo-US Science and Technology Forum, New Delhi and the National Science Foundation, USA, for their support, without which the meeting could not have taken place. In particular, two individuals, Drs Radhakrishna Pillai and Satish Mundayoor, both from the Rajiv Gandhi Centre, Trivandrum, need to be singled out for having gone out of their way to ensure that the venue and local arrangements were perfect.

Vidyanand Nanjundiah* and Stuart A Newman**

*Developmental Biology and Genetics Laboratory, Indian Institute of Science, Bangalore 560 012, India

(Email,vidya@ces.iisc.ernet.in)

**Department of Cell Biology and Anatomy, Basic Sciences Building, New York Medical College, Valhalla, New York 10595, USA

(Email,newman@nymc.edu) 